Trinity University

Digital Commons @ Trinity

Mathematics Faculty Research

Mathematics Department

$12-2007$

\title{
On the Asymptotic Stability of Linear Volterra Difference Equations of Convolution Type
}

Saber Elaydi

Trinity University, selaydi@trinity.edu

E Messina

A Vecchio

Follow this and additional works at: https://digitalcommons.trinity.edu/math_faculty

Part of the Mathematics Commons

\section{Repository Citation}

Elaydi, S., Messina, E., \& Vecchio, A. (2007). On the asymptotic stability of linear Volterra difference equations of convolution type. Journal of Difference Equations and Applications, 13(12), 1079-1084. doi:10.1080/10236190701264529

This Post-Print is brought to you for free and open access by the Mathematics Department at Digital Commons @ Trinity. It has been accepted for inclusion in Mathematics Faculty Research by an authorized administrator of Digital Commons @ Trinity. For more information, please contact jcostanz@trinity.edu. 


\title{
A note on the asymptotic stability of linear Volterra difference equations of convolution type
}

\author{
S. Elaydi \\ Department of Mathematics \\ Trinity University - San Antonio, Texas 78212, USA \\ selaydi@trinity.edu \\ E. Messina \\ Dipartimento di Matematica e Applicazioni, \\ Università degli Studi di Napoli "Federico II"- Via Cintia, I-80126 Napoli, Italy \\ eleonora.messina@unina.it \\ A. Vecchio \\ Ist. per Appl. del Calcolo "M.Picone", Sede di Napoli - CNR - \\ Via P. Castellino,111 - 80131 Napoli - Italy \\ a.vecchio@iac.cnr.it
}

\begin{abstract}
We show that the condition $|a|+\left|\sum_{l=0}^{+\infty} b_{l}\right|<1$ is not necessary, though sufficient, for the asymptotic stability of $x_{n+1}=a x_{n}+\sum_{l=0}^{+\infty} b_{n-l} x_{l}$. We prove the existence of a class of Volterra difference equations that violate this condition but whose zero solutions are asymptotically stable.
\end{abstract}

Keywords: Volterra difference equations, asymptotic stability, convolution, open question. 2000 Mathematics Subject Classification: 34D05, 34D20, 39A11 


\section{Introduction}

Let us consider the linear convolution Volterra Difference Equations (VDEs)

$$
x_{n+1}=a x_{n}+\sum_{l=0}^{n} b_{n-l} x_{l}, n \geq 0,
$$

where $x_{0}$ is given and $a \in \mathbb{R}$, and recall the following definitions of stability [8, p.176].

Definition 1 The zero solution of (1.1) is said to be

1. stable if for all $\epsilon>0$ there exists $\delta(\epsilon)>$ such that $\left|x_{0}\right|<\delta$ implies $\left|x_{n}\right|<\epsilon$ for all $n \geq 0$;

2. asymptotically stable if it is stable and there exists $\mu$ such that $\left|x_{0}\right|<\mu$ implies $\lim _{n \rightarrow+\infty} x_{n}=0$.

In the last two decades, many authors investigated the asymptotic stability of (1.1) [8, p.39] and $[2,3,5,6,7,9,10,12]$ mostly by means of the $Z$-transform or the Liapunov

approach [8, chap.6]. The earliest thorough study of (1.1) was carried out by Elaydi in [5]. However, the condition he proved, as he himself writes, although necessary and sufficient, is not "practical" because it requires the localization of the roots of a complex function related to the $Z$-transform of the sequence $\left\{b_{n}\right\}_{n \geq 0}$ of the coefficients of (1.1).

In the same paper the following explicit criterion for the asymptotic stability of (1.1) was provided:

Theorem 1.1 [5, 8] Suppose that $b_{n}$ does not change sign for $n \geq 0$ and

$$
|a|+\left|\sum_{l=0}^{+\infty} b_{l}\right|<1,
$$

then the zero solution of (1.1) is asymptotically stable.

This is a nice sufficient condition directly expressed in terms of the coefficients of the VDE considered and, until now, it is still an open question whether or not (1.2) is also necessary for the asymptotic stability of (1.1) [8, p.296].

The purpose of this paper is to answer this question. Namely, we prove that, starting from any sequence $\left\{\beta_{n}\right\}_{n \geq 0}$ satisfying $(-1)^{i} \Delta \beta_{n} \leq 0, \quad i=0,1,2$, we can construct an infinite number of VDEs of the type (1.1) whose solution is asymptotically stable and whose coefficients satisfy $|a|+\left|\sum_{l=0}^{+\infty} b_{l}\right| \geq 1$. Hence, we can conclude that (1.2) is not necessary.

In the next section we prove our result by effectively constructing a class of asymptotically stable VDEs that volate (1.2). 


\section{Stability of VDEs}

In this section we describe our result on the stability of the zero solution of (1.1). Since our approach in the study of the asymptotic properties of $x_{n}$ will go through the Liapunov technique for VDEs, we refer to [1] and we report here the main result for the general Volterra difference equation of unbounded order

$$
y_{n+1}=F\left(n, y_{0}, \ldots, y_{n}\right), n \geq 0, y_{n} \in \mathbb{R},
$$

with $F(n, 0, \ldots, 0)=0$.

Theorem 2.1 Let $\omega_{i}(r), r \in \mathbb{R}, i=1,2$, be scalar continuous increasing functions such that $\omega_{i}(0)=0, i=1,2$. If there exists a scalar function $V\left(n, y_{0}, \ldots, y_{n}\right)$, continuous with respect to all the variables $y_{0}, y_{1}, \ldots, y_{n}, \ldots$, such that

a) $V(0,0)=0$

b) $V\left(n, y_{0}, \ldots, y_{n}\right) \geq \omega_{1}\left(\left|y_{n}\right|\right), \quad n \geq 0$

c) $\Delta V_{n}=V\left(n+1, y_{0}, \ldots, y_{n}, F\left(n, y_{0}, \ldots, y_{n}\right)\right)-V\left(n, y_{0}, \ldots, y_{n}\right) \leq 0, \quad n \geq 0$

then the solution of (2.1) is stable. If, in addition

d) $\Delta V_{n} \leq-\omega_{2}\left(\left|y_{n}\right|\right)$

then the solution of (2.1) is asymptotically stable.

The following theorem shows how to obtain a class of VDEs which is asymptotically stable even though (1.2) is not satisfied.

Theorem 2.2 Assume there exists a sequence $\left\{b_{n}\right\}_{n \in \mathbb{N}}$ that satisfies

i. $(-1)^{k} \Delta^{k} b_{n} \leq 0, k=0,1,2$, for each $n=0,1, \ldots$.

ii. $b_{1}-2 b_{0}-2<0$

and one of the following two conditions holds

iiii $_{1} \cdot b_{1}+2 b_{0}+1 \geq 0, b_{1}<0$

iii $_{2} \cdot b_{1}+4 b_{0}+2>0$,

then it is always possible to find $a \in \mathbb{R}$ such that $|a|+\left|\sum_{l=0}^{\infty} b_{l}\right| \geq 1$ and the zero solution of $x_{n+1}=a x_{n}+\sum_{l=0}^{\infty} b_{n-l} x_{l}$ is asymptotically stable.

Proof. Let $b_{n}$ be such a sequence and choose $a$ such that

$$
\frac{b_{1}-2 b_{0}-2}{2}<a \leq \min \left\{-\left(1+b_{0}\right), b_{1}+b_{0}\right\} \text {. }
$$


With this form for $b_{n}$ and the values prescribed in (2.2) for $a$, it is obvious that $|a|+$ $\left|\sum_{l=0}^{+\infty} b_{l}\right| \geq 1$ and hence (1.2) is not satisfied. Let us consider the Liapunov function

$$
\begin{aligned}
V\left(n, x_{0}, \ldots, x_{n}\right)=- & \left(b_{n}+a \delta_{n, 0}\right)\left(\sum_{j=0}^{n} x_{j}\right)^{2} \\
& +\left(b_{0}+a+2\right) x_{n}^{2}+\sum_{i=0}^{n-1}\left(b_{n-i}-b_{n-1-i}-a \delta_{n-1, i}\right)\left(\sum_{j=0}^{n} x_{j}\right)^{2}
\end{aligned}
$$

where $\delta_{i, j}=0$ if $i \neq j$ and $\delta_{i, i}=1$, and set

$$
\begin{aligned}
& \omega_{1}(y)=\left(b_{0}+a+2\right) y^{2} \\
& \omega_{2}(y)=-\left(b_{1}-2\left(b_{0}+a\right)-2\right) y^{2} .
\end{aligned}
$$

$V$ is continuous with respect to $x_{0}, \ldots, x_{n}$ and $\omega_{1}$ and $\omega_{2}$ are continuous. Notice that from Conditions i and ii, we have $b_{1}>-2$. This implies using (2.2) that $b_{0}+a+2>0$, and $b_{1}-2\left(b_{0}+a\right)-2<0$. Hence, $\omega_{1}(y)$ and $\omega_{2}(y)$ in $(2.4)$ are positive increasing functions. Now we show that $V$ satisfies hypotheses a)-d) of Theorem 2.1.

Of course, it is clear from $(2.3)$ that $V(0,0)=0$ and

$$
V\left(n, x_{0}, \ldots, x_{n}\right) \geq \omega_{1}\left(\left|x_{n}\right|\right)
$$

In order to prove that $V$ satisfies c), let us consider

$$
\begin{gathered}
\Delta V_{n}=V\left(n+1, x_{0}, x_{1}, \ldots, x_{n+1}\right)-V\left(n, x_{0}, x_{1}, \ldots, x_{n}\right) \\
=-\left(b_{n+1}+a \delta_{n+1,0}\right)\left(\sum_{j=0}^{n+1} x_{j}\right)^{2}+\left(b_{0}+a+2\right) x_{n+1}^{2} \\
\quad+\sum_{i=0}^{n}\left(b_{n+1-i}-b_{n-i}-a \delta_{n, i}\right)\left(\sum_{j=i}^{n+1} x_{j}\right)^{2}-V\left(n, x_{0}, \ldots, x_{n}\right) \\
=-\left(b_{n+1}+a \delta_{n+1,0}\right)\left(\left(\sum_{j=0}^{n} x_{j}\right)^{2}+x_{n+1}^{2}+2 x_{n+1} \sum_{j=0}^{n} x_{j}\right)+\left(b_{0}+a+2\right) x_{n+1}^{2} \\
+\sum_{i=0}^{n}\left(b_{n+1-i}-b_{n-i}-a \delta_{n, i}\right)\left(\left(\sum_{j=i}^{n} x_{j}\right)^{2}+x_{n+1}^{2}+2 x_{n+1} \sum_{j=i}^{n} x_{j}\right) \\
-V\left(n, x_{0}, x_{1}, \ldots, x_{n}\right), \quad n \geq 0 .
\end{gathered}
$$


By manipulating this expression we get:

$$
\begin{aligned}
& \Delta V_{n}=-\left(b_{n+1}+a \delta_{n+1,0}\right)\left(\sum_{j=0}^{n} x_{j}\right)^{2}-\left(b_{n+1}+a \delta_{n+1,0}\right)\left(x_{n+1}^{2}+2 x_{n+1} \sum_{j=0}^{n} x_{j}\right) \\
& +\left(b_{0}+a+2\right) x_{n+1}^{2}+\sum_{i=0}^{n}\left(b_{n+1-i}-b_{n-i}-a \delta_{n, i}\right) x_{n+1}^{2} \\
& +2 x_{n+1} \sum_{i=0}^{n}\left(b_{n+1-i}-b_{n-i}-a \delta_{n, i}\right) \sum_{j=i}^{n} x_{j}+\sum_{i=0}^{n}\left(b_{n+1-i}-b_{n-i}-a \delta_{n, i}\right)\left(\sum_{j=i}^{n} x_{j}\right)^{2} \\
& +\left(b_{n}+a \delta_{n, 0}\right)\left(\sum_{j=0}^{n} x_{j}\right)^{2}-\left(b_{0}+a+2\right) x_{n}^{2}-\sum_{i=0}^{n-1}\left(b_{n-i}-b_{n-1-i}-a \delta_{n-1, i}\right)\left(\sum_{j=i}^{n} x_{j}\right)^{2} \\
& =B_{n}-\left(b_{n+1}+b_{n}-a \delta_{n, 0}\right)\left(\sum_{j=0}^{n} x_{j}\right)^{2} \\
& +\sum_{i=0}^{n-1}\left(b_{n+1-i}-2 b_{n-i}+b_{n-1-i}+a \delta_{n-1, i}\right)\left(\sum_{j=i}^{n} x_{j}\right)^{2}+\left(b_{1}-2\left(b_{0}+a\right)-2\right) x_{n}^{2}, \quad n \geq 0,
\end{aligned}
$$

where

$$
\begin{aligned}
B_{n}= & 2 x_{n+1}\left(\sum_{i=0}^{n}\left(b_{n+1-i}-b_{n-i}-a \delta_{n, i}\right) \sum_{j=i}^{n} x_{j}-\left(b_{n+1}+a \delta_{n+1,0}\right) \sum_{j=0}^{n} x_{j}\right) \\
& +x_{n+1}^{2}\left(-\left(b_{n+1}+a \delta_{n+1,0}\right)+\left(b_{0}+a+2\right)+\sum_{i=0}^{n}\left(b_{n+1-i}-b_{n-i}-a \delta_{n, i}\right)\right) .
\end{aligned}
$$

By interchanging the order of summation in $\sum_{i=0}^{n}\left(b_{n+1-i}-b_{n-i}-a \delta_{n, i}\right) \sum_{j=i}^{n} x_{j}$, it is easy to prove that

$$
\begin{aligned}
B_{n}= & 2 x_{n+1}\left(\sum_{j=0}^{n} x_{j}\left(\sum_{i=0}^{j}\left(b_{n+1-i}-b_{n-i}\right)-\sum_{i=0}^{j} a \delta_{n, i}-b_{n+1}\right)\right) \\
& +x_{n+1}^{2}\left(-\left(b_{n+1}+b_{0}+a+2\right)+\sum_{i=0}^{n}\left(b_{n+1-i}-b_{n-i}\right)-a\right),
\end{aligned}
$$

and by using the fact that $\sum_{i=0}^{j}\left(b_{n+1-i}-b_{n-i}\right)=b_{n+1}-b_{n-j}$, it comes out that $B_{n}=0$. For the other quantities involved in the expression of $\Delta V_{n}$, we have that

$$
b_{n+1}+b_{n}-a \delta_{n, 0}= \begin{cases}b_{1}+b_{0}-a & \text { if } n=0 \\ b_{n+1}+b_{n} & \text { if } n>0\end{cases}
$$

and

$$
b_{n+1-i}-2 b_{n-i}+b_{n-1-i}-a \delta_{n-1, i}= \begin{cases}b_{2}-2 b_{1}+b_{0}+a & \text { if } i=n-1 \\ b_{n+1-i}-2 b_{n-i}+b_{n-1-i} & \text { otherwise }\end{cases}
$$


As a consequence of the hypotheses on $b_{n}$ and of our choice (2.2) for $a$, both (2.5) and (2.6) are less than or equal to zero. Therefore,

$$
\Delta V_{n} \leq-\omega_{2}\left(\left|x_{n}\right|\right), n=0,1, \ldots,
$$

where $\omega_{2}(|\cdot|)$ is given in $(2.4)$.

In conclusion, $V$ satisfies all the hypotheses of Theorem 2.1, and consequently, the zero solution of (1.1) is asymptotically stable.

Notice that the result in theorem (2.2) is valid subject to the existence of a sequence $b_{n}$ which satisfies Conditions i, ii, and either $\mathrm{iii}_{1}$ or $\mathrm{iii}_{2}$. The following theorem provides an algorithm on how to construct such a sequence.

Theorem 2.3 There exists an infinite number of sequences $\left\{b_{n}\right\}_{n \in \mathbb{N}}$ such that

i. $(-1)^{k} \Delta^{k} b_{n} \leq 0, k=0,1,2, n \geq 0$

ii. $b_{1}-2 b_{0}-2<0$

iii $_{2} \cdot b_{1}+4 b_{0}+2>0$,

hold.

Proof. Let $\left\{\beta_{n}\right\}_{n \in \mathbb{N}}$ be a sequence whose terms satisfy $(-1)^{k} \Delta^{k} \beta_{n} \leq 0$, for $k=0,1,2$ and $n \geq 0$ (for instance $\beta_{n}=-\frac{1}{2^{n}}$ ). Set $b_{n}=-\frac{\beta_{n}}{\theta \beta_{0}}$ where $\theta>2+\frac{\beta_{1}}{\beta_{0}}$. From the assumptions on $\beta_{n}$, it immediately follows that $b_{n} \leq 0$, for $n \geq 0, b_{n}-b_{n-1} \geq 0$ and $b_{n+1}-2 b_{n}+b_{n-1} \leq 0$, for all $n \geq 1$ and then i. holds. Now consider

$$
b_{1}-2 b_{0}-2=\frac{-\beta_{1}-2(\theta-1) \beta_{0}}{\theta \beta_{0}}
$$

and

$$
b_{1}+4 b_{0}+2=\frac{-\beta_{1}-2(2-\theta) \beta_{0}}{\theta \beta_{0}} .
$$

Since $\beta_{0}$ and $\beta_{1}$ are negative and $\theta>2+\frac{\beta_{1}}{\beta_{0}}$, the first expression above is negative, while the second is positive. Hence, ii. and $\mathrm{iii}_{2}$. hold. The result stated in the theorem is then proved.

Example 2.4 It is easy to check that the sequence $\left\{b_{n}\right\}_{n \in \mathbb{N}}$ with $b_{n}=-\frac{1}{3} \frac{1}{2^{n}}$ satisfies the hypotheses of Theorem 2.2. In this case, any a belonging to $\left(-\frac{3}{4},-\frac{2}{3}\right)$ leads to the asymptotic stability of (1.1). 


\section{Concluding remarks}

In this note we propose a constructive analysis on the asymptotic stability of a class of VDEs of convolution type. In particular we show the non necessity of condition (1.2) for the asymptotic stability of (1.1) by proving the existence of infinite sequences $\left\{b_{n}\right\}_{n \in \mathbb{N}}$ which, for certain values of $a$, lead to the asymptotic stability of (1.1) and that, all the same, don't fulfill (1.2). The problem of finding "easily verifiable" necessary and sufficient condition for the asymptotic stability of the zero solution of (1.1) remain therefore an open question!

\section{References}

[1] M.R. Crisci, V. B. Kolmanovskii, E. Russo and A. Vecchio, Stability of continuous and discrete Volterra integro-differential equations by Liapunov approach, J. Integral Equations Appl. 7 (1995), no. 4, 393-411.

[2] M.R. Crisci, V. B. Kolmanovskii, E. Russo and A.Vecchio, Stability of difference Volterra equations: direct Liapunov method and numerical procedure. Advances in difference equations, II. Comput. Math. Appl. 36 (1998), no. 10-12, 77-97.

[3] M.R. Crisci, V.B. Kolmanovskii, E. Russo, A. Vecchio, Stability of discrete Volterra equations of Hammerstein type. J. Differ. Equations Appl. 6 (2000), no. 2, 127-145.

[4] M.R. Crisci, V. B. Kolmanovskii, E. Russo and A. Vecchio, Stability of continuous and discrete Volterra integro-differential equations by Liapunov approach, J. Integral Equations Appl., 7 (2004), no. 4, 393-411.

[5] S. Elaydi, Stability of Volterra difference equations of convolution type. Dynamical systems, 66-72, Nankai Ser. Pure Appl. Math. Theoret. Phys., 4, World Sci. Publ., River Edge, NJ, 1993.

[6] S. Elaydi, Periodicity and stability of linear Volterra difference systems, J. Math. Anal. Appl., 181 (1994), no. 2, 483-492.

[7] S. Elaydi, Global stability of difference equations. World Congress of Nonlinear Analysts '92, Vol. I-IV , 1131-1138, de Gruyter, Berlin, 1996.

[8] S. Elaydi, An Introduction To Difference Equations, Third edition. Springer, New York, 2005

[9] S. Elaydi, S. Murakami, Asymptotic stability versus exponential stability in linear Volterra difference equations of convolution type, J. Differ. Equations Appl. 2 (1996), no. $4,401-410$.

[10] C. Lubich, On the stability of linear multistep methods for Volterra convolution equations, IMA J. Numer. Anal. 3 (1983), no. 4, 439-465.

[11] A. Vecchio, Stability of backward differentiation formulas for Volterra integrodifferential equations. J. Comput. Appl. Math. 115 (2000), no. 1-2, 565-576. 
[12] A. Vecchio, Stability results on some direct quadrature methods for Volterra integrodifferential equations, Dynam. Systems Appl. 7 (1998), no. 4, 501-518. 